\title{
Neem-silicone lotion and ultrasound nit comb: a randomised, controlled clinical trial treating head louse infestation
}

\author{
Ian F. Burgess", Elizabeth R. Brunton, Nazma A. Burgess and Mark N. Burgess
}

\begin{abstract}
Background: A neem-based conditioner lotion (ONC) used against head lice was previously tested clinically and shown to be relatively ineffective. To improve the activity against lice and their eggs, it was reformulated into a silicone vehicle (NNC) as part of a project to improve pediculicidal medical devices. An ultrasound nit comb was also developed to be used in conjunction with the neem lotion to remove louse eggs from the hair.

Methods: A single-centre, parallel group, randomised, controlled, open-label community-based clinical study was set up to test the NNC lotion in comparison with a marketed product based on isopropyl myristate and cyclomethicone (IPM/C) with two treatments 7 days apart. In parallel, the lubrication effects of the NNC and ONC lotions were compared in use with the ultrasound comb.

Results: For 134 randomised participants (50 NNC, 53 IPM/C, 17 NNC plus comb, and 14 ONC plus comb), the cure rate was $72.0 \%$ for NNC and $69.8 \%$ for IPM/C (OR 1.112, 95\% Cl, 0.47 to 2.61). Additional combing gave $82.4 \%$ cure using NNC and 28.6\% using ONC (OR 11.67; 95\% Cl 2.13 to 64.04). No difference in efficacy of nit removal was detected although combing with conditioner was physically easier.
\end{abstract}

Conclusions: One percent neem oil in silicone (NNC) is as effective to eliminate head lice as other silicone products. Nit combing is easier using a conditioner lubricant to facilitate sliding of the eggshells along hairs.

Trial registration: Current Controlled Trials Registry ISRCTN77673809

Keywords: Pediculosis capitis, Treatment, Silicone, Neem oil, Nit combing, Ultrasound

\section{Background}

Consumer preference to use natural treatments to eliminate head lice has increased with the spread of resistance to insecticides. Apart from essential oils (Grieve et al. 2007; Burgess et al. 2010; Barker and Altman 2010), few plant products have undergone clinical trials. The most widely used is oil from the neem tree, Azadirachta indica A. Juss (Meliaceae), which contains a number of putatively pharmacologically active triterpenoids. One shampoo from Germany based on a concentrated extract has undergone investigations in villages in Egypt and Arabia (Heukelbach et al. 2006; Abdel-Ghaffar and Semmler 2007; Schmahl et al. 2010; Mehlhorn et al. 2011; Abdel-Ghaffar et al. 2012) with

\footnotetext{
* Correspondence: ian@insectresearch.com

Medical Entomology Centre, Insect Research \& Development Limited, 6 Quy Court, Colliers Lane, Stow-cum-Quy, Cambridge CB25 9AU, UK
}

reported high efficacy following a single application. In contrast, an alcohol-based Australian product, containing $6 \%$ neem oil and $16 \%$ eucalyptus, required two applications plus combing in a Thai study (Thawornchaisit et al. 2012). However, both results contrast strongly with our earlier low success using a neem oil-based conditioner plus combing (Brown and Burgess 2017).

This work formed part of a European Commission Sixth Framework Craft project to develop new medical devices to treat head louse infestation. Previously, we described a laboratory evaluation of the ultrasound comb, developed as part of this investigation, for facilitation of nit removal (Burgess et al. 2016). In parallel, the neem oil-based lotion was reformulated to improve the activity against both lice and their eggs. It was hoped the changes would also improve the lubrication characteristics for nit removal. 
These newly developed class I medical devices were investigated in a randomised controlled clinical study.

\section{Methods \\ Setting}

This randomised, controlled comparison of two pediculicide treatments and two combing plus combing-aid treatments was a single-site study conducted in and around Cambridge, UK. Participants were recruited through local advertising. Each household received an information booklet, and an investigator arranged a domiciliary visit. All household members were screened for lice using a plastic detection comb and followed eligibility criteria used in previous studies (Burgess et al. 2007). Exclusions were treatment for lice within 2 weeks; sensitivity to treatment components or long-term scalp conditions other than lice; using hair bleach, dyes, or permanent waves; or treatments with trimethoprim products within 4 weeks. We also excluded pregnant and breast feeding females, previous participation in this study, or other studies within 4 weeks. Age eligibility ranged from 2 years with no upper limit. Infested non-participants were offered a standard of care treatment (4\% dimeticone lotion) to minimise reinfestation of participants. No payment was offered for participation.

Enrolment was planned at 176 participants across four treatment groups: 66 using reformulated Nice 'n Clear lotion (NNC), 66 50:50 isopropyl myristate in cyclomethicone (IPM/C) (Full Marks solution), 22 original formulation Nice ' $\mathrm{n}$ Clear head lice lotion (ONC) plus combing using the ClearBrush ${ }^{\circ}$ ultrasound nit comb (CB), and 22 NNC plus the ClearBrush ${ }^{\circ}$ ultrasonic nit comb. Following manufacturing difficulties for the ultrasound comb, the study was terminated early to comply with European Commission deadline rules for project completion. At premature termination, 134 participants had been treated and followed up: $50 \mathrm{NNC}, 53 \mathrm{IPM} / \mathrm{C}, 14 \mathrm{ONC}$ plus ClearBrush $^{\circ}$, and $17 \mathrm{NNC}$ plus ClearBrush ${ }^{\circ}$.

A total of 136 people from 75 households (112 children and 24 adults) were consented between 13 September 2007 and 25 March 2008. Other household members and four other families were screened but had no lice, and two consenting participants were eliminated from analyses for a protocol violation before the first treatment. There were two withdrawals from each lotion group (Fig. 1); seven others missed one or more assessments. Therefore, the intention to treat (ITT) population analysed was 134 and the per-protocol (PP) population 125.

\section{Treatments}

All treatments were given on day 0 and repeated on day 7. Four treatment products were used:

1. Investigative product: a new formulation containing 1\% neem seed oil, dimeticone PEG-PPG co-polymer, and cyclomethicone-5 (reformulated Nice 'n Clear lotion or "New Nice "n Clear" lotion (NNC), Nelsons, Wimbledon, UK)

2. Comparator product: a 50:50 mixture of isopropyl myristate with cyclomethicone (Full Marks solution (IPM/C), Reckitt Benckiser, Slough, UK)

3. Combing aid: the original $1 \%$ neem oil conditioner rinse (original formulation Nice ' $n$ Clear head lice lotion (ONC), Nelsons, Wimbledon, UK) containing $1 \%$ neem seed oil, tea tree oil, lavender oil, and other herbal extracts

4. Nit comb: a newly designed comb activated by ultrasound (ClearBrush ${ }^{\circ}$, Nelsons, Wimbledon, UK) (Burgess et al. 2016), which was the primary development objective of the whole project

$\mathrm{NNC}$ and IPM/C were compared for efficacy against head lice when applied to dry hair until saturated. NNC was left in place overnight and then washed off with shampoo. IPM/C was applied for 10 min before washing off with shampoo. All participants used the same shampoo, supplied by the investigators.

The lubricant effects of $\mathrm{NNC}$ and $\mathrm{ONC}$ were compared during nit removal using the ClearBrush ${ }^{\circ}$. This device (Fig. 2) delivered ultrasound to the comb teeth through two piezoelectric actuators, as described previously (Burgess et al. 2016). A fresh standard comb tooth unit (Innomed ${ }^{\mathrm{m}}$ comb, Hogil Pharmaceutical Corp., White Plains, NY, USA) was fitted for each participant to eliminate any risk of cross-contamination. Before the clinical work, the two lubricants were compared in the laboratory for their effect on loosening louse eggs (peak force) and sliding them along hair (average force), using a slip-peel tester (SP-2000, IMASS, Inc., Accord, MA, USA) as described previously (Burgess et al. 2016; Burgess 2010).

NNC was applied to dry hair but ONC to pre-washed and towel-dried hair. After thorough application, the hair was combed systematically using the ClearBrush ${ }^{\circ}$ comb with ultrasound switched on. Louse eggs and nits removed during combing were recovered for later examination and counting in the laboratory. The use of other nit combs or treatment products was not permitted during the course of the study.

\section{Outcome measures}

Follow-up assessments were made in all groups on days 2, 6, 9, and 14 using a plastic detection comb ("PDC" comb, KSL Consulting ApS, Helsinge, Denmark). Any lice recovered were fixed into the case documentation as a permanent record. The primary outcome measure was the elimination of infestation after completing treatment, i.e. no lice at days 9 and 14. Outcomes were classified as cure, reinfestation after cure, or treatment failure. 


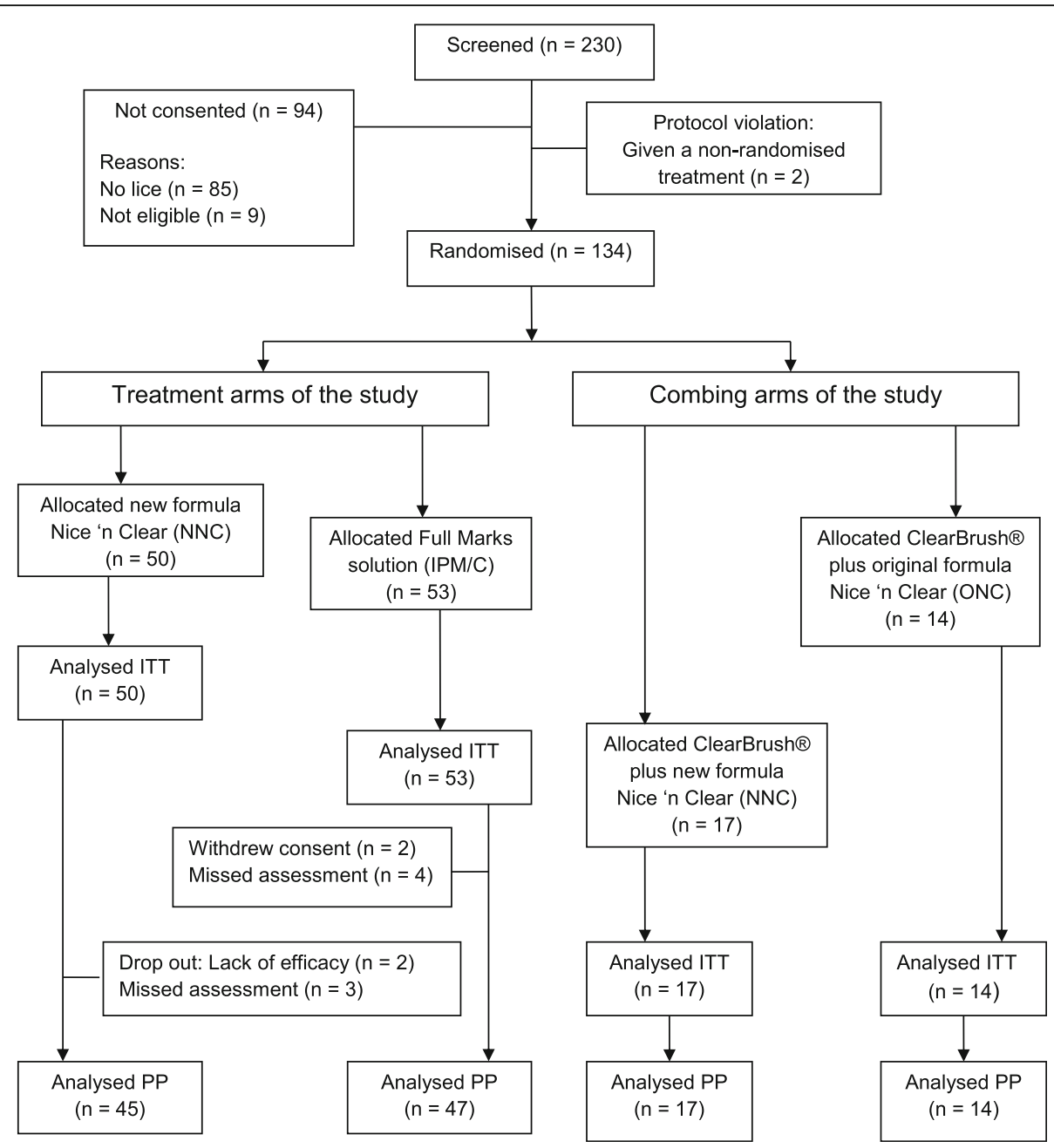

Fig. 1 Flowchart of participant progress through the study. The flowchart shows the numbers of participants in each of the randomised study groups and their progress through the treatment phase of the study. All participants were treated on day 0 and day 7 and assessments of outcome were made on days 2, 6, 9, and 14 (not shown on flowchart)

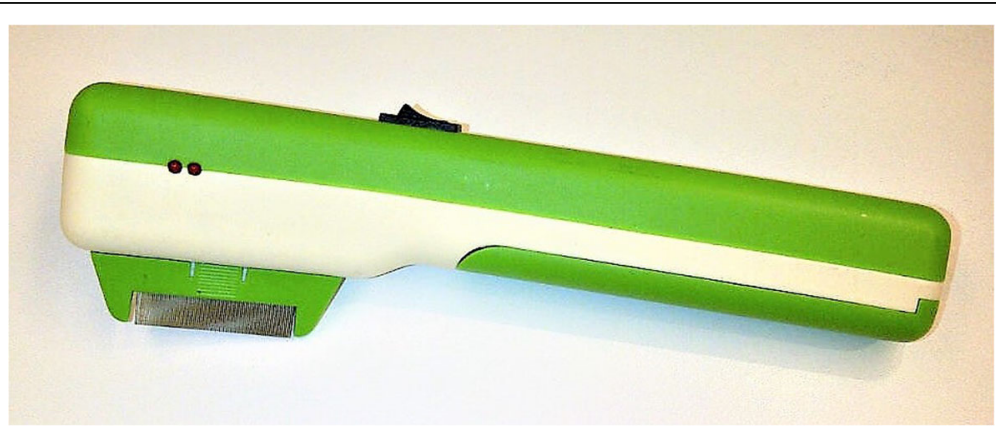

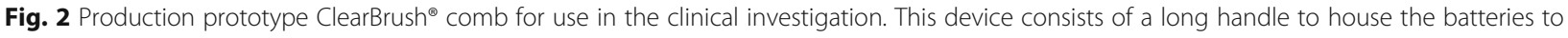
power the ultrasound actuators. The combing head has a detachable section for changing the steel comb tooth units and has tell-tale lights for ultrasound generation and battery power, actuated by an on/off switch. The comb head is angled relative to the handle by $16.5^{\circ}$ 


\section{Sample size}

It was estimated that equivalence for the lotions to within 25\% could be identified using 132 participants (66 treated with NNC and 66 with IPM/C) with 90\% power and 95\% confidence, assuming an underlying success rate for $\mathrm{NNC}$ of $75 \%$ based on ex vivo data. The premature termination and lower efficacy outcomes reduced the power of the study for identification of $25 \%$ equivalence with $95 \%$ confidence to $75 \%$.

For the comparison of lubricants using the ClearBrush $^{\oplus}$ ultrasound comb to remove nits, we could make no clear estimation of the required number of participants based on expectations of outcome because there were no appropriate prior data to work from comparing different combs or combing techniques. Only one broadly similar investigation was found, which used 22 participants per group, so the study was structured on that basis (de Souza et al. 2001).

\section{Randomisation and blinding}

Treatment instructions in sealed, opaque, numbered envelopes randomised using an online computer generated list (Dallal 2007) were distributed to investigators in balanced blocks of eight. Participants were allocated treatment using the next available numbered envelope held by the investigator. Post-treatment assessments were performed by different investigators from those involved in treatment so that they remained blind to the treatment allocation.

It became necessary to generate a two-stage randomisation because usable models of the ClearBrush ${ }^{\oplus}$ could not be manufactured in time for the study initiation. Initially, half of the participants were randomised only between the two lotion treatments (NNC and IPM/C).

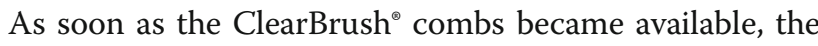
randomisation sequence was recalculated to include all four treatment groups.

\section{Statistical analysis}

We conducted analyses based on both the ITT and PP populations. Differences in success rates were measured by the $95 \%$ confidence interval calculated using a normal approximation to the binomial distribution. Comparison of groups in baseline characteristics, safety, acceptability, and efficacy were tested using Fisher's exact test for yes/no variables and the Mann-Whitney $U$ test for ranked variables.

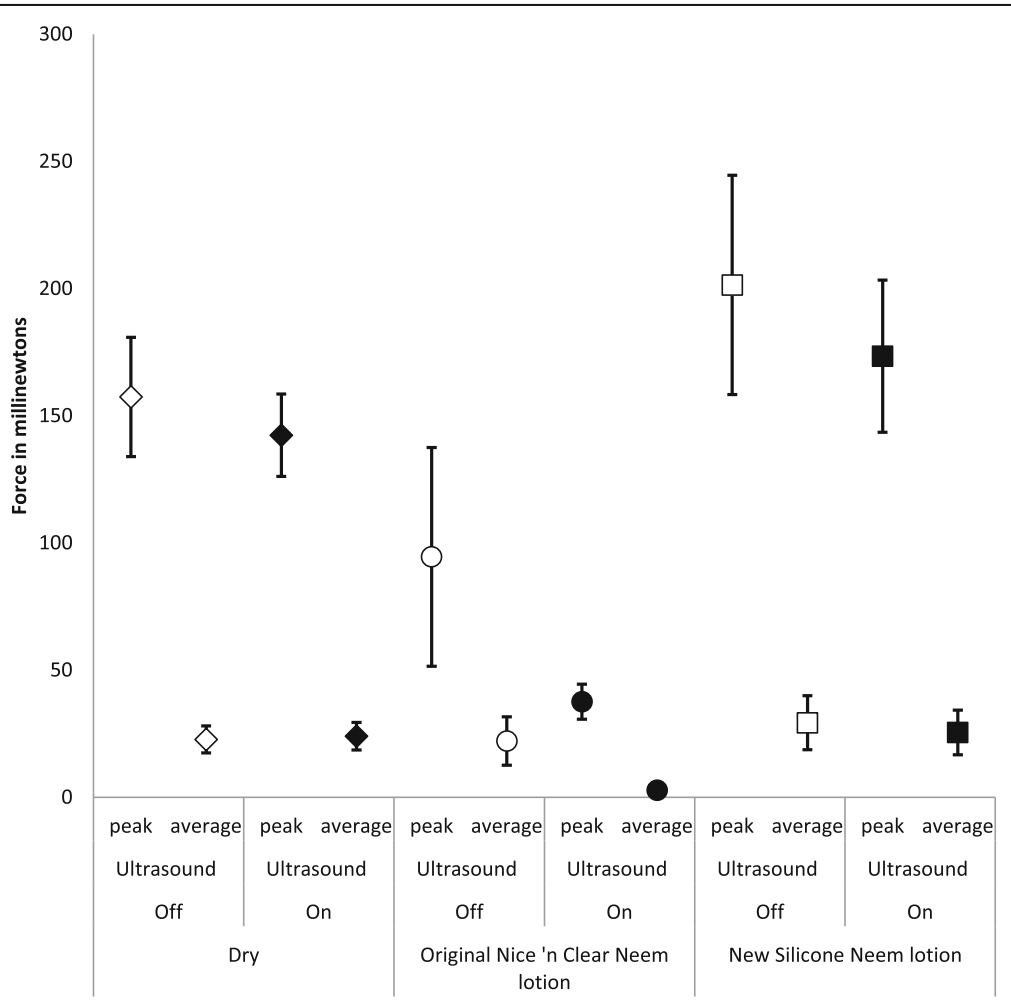

Fig. 3 Comparison of peak and average force required to remove louse eggs using the NNC and ONC products as lubricants using a slip-peel tester to measure forces. White symbols indicate the forces generated when no ultrasound was applied; black symbols show the same forces under the influence of ultrasound. Each dataset was generated using a minimum of 20 louse eggs on human hairs 
Table 1 Demographic characteristics of the intention to treat population at baseline

\begin{tabular}{|c|c|c|c|c|c|c|}
\hline \multicolumn{2}{|l|}{ Characteristic } & NNC lotion & IPM/C lotion & $\mathrm{NNC}+\mathrm{CB}$ & $\mathrm{ONC}+\mathrm{CB}$ & Total \\
\hline \multicolumn{2}{|c|}{ Number of participants } & 50 & 53 & 17 & 14 & 134 \\
\hline \multirow[t]{5}{*}{ Age } & $2-6$ & $12(24.0 \%)$ & $11(20.8 \%)$ & $4(23.5 \%)$ & $2(14.3 \%)$ & $29(21.6 \%)$ \\
\hline & $7-9$ & $19(38.0 \%)$ & 15 (28.9\%) & $4(23.5 \%)$ & $7(50.0 \%)$ & 45 (33.6\%) \\
\hline & $10-16$ & $8(16.0 \%)$ & $16(30.2 \%)$ & $7(41.2 \%)$ & $5(35.7 \%)$ & $36(26.9 \%)$ \\
\hline & $>17$ & $11(22.0 \%)$ & $11(20.8 \%)$ & $2(11.8 \%)$ & $0(0.0 \%)$ & $24(17.9 \%)$ \\
\hline & Median & 9 & 10 & 10 & 9 & 9 \\
\hline Sex & Female & $38(76.0 \%)$ & $43(81.1 \%)$ & $12(70.6 \%)$ & $9(64.3 \%)$ & $102(76.1 \%)$ \\
\hline \multirow[t]{2}{*}{ Infestation } & Light & $33(66.0 \%)$ & 31 (58.5\%) & $10(58.8 \%)$ & $10(71.4 \%)$ & $84(62.7 \%)$ \\
\hline & Heavy & $4(8.0 \%)$ & $8(15.1 \%)$ & 1 (5.9\%) & $0(0.0 \%)$ & $13(9.7 \%)$ \\
\hline \multicolumn{7}{|c|}{ Hair characteristics } \\
\hline \multirow[t]{2}{*}{ Length } & Above ears & $12(24.0 \%)$ & $8(15.1 \%)$ & $3(17.7 \%)$ & $4(28.6 \%)$ & $27(20.2 \%)$ \\
\hline & Below shoulders & 27 (54.0\%) & 32 (60.4\%) & $10(58.8 \%)$ & $8(57.1 \%)$ & 77 (57.5\%) \\
\hline \multirow[t]{2}{*}{ Thickness } & Fine/medium & $36(72.0 \%)$ & $28(52.8 \%)$ & $11(64.7 \%)$ & $11(78.6 \%)$ & $86(64.2 \%)$ \\
\hline & Thick & $14(28.0 \%)$ & 25 (47.2\%) & $6(35.3 \%)$ & $3(21.4 \%)$ & $48(35.8 \%)$ \\
\hline \multirow[t]{2}{*}{ Curl } & Straight & $31(62.0 \%)$ & 38 (71.7\%) & $14(82.4 \%)$ & $5(35.7 \%)$ & $88(65.7 \%)$ \\
\hline & Wavy/curly & $19(38.0 \%)$ & $15(28.3 \%)$ & $3(17.7 \%)$ & $9(64.3 \%)$ & $46(34.3 \%)$ \\
\hline Type & Normal & 49 (98.0\%) & $51(96.2 \%)$ & $17(100 \%)$ & $14(100 \%)$ & $131(97.8 \%)$ \\
\hline \multicolumn{2}{|c|}{ Other family member in study } & $40(80.0 \%)$ & $44(83.0 \%)$ & $14(82.4 \%)$ & $12(85.7 \%)$ & $110(82.1 \%)$ \\
\hline
\end{tabular}

\section{Results}

\section{Laboratory combing comparison}

The laboratory comparison of the two neem-based lubricants plus the ClearBrush ${ }^{\circ}$ ultrasound comb found the silicone-based NNC was a less-effective lubricant than the conditioner-based $\mathrm{ONC}$, irrespective of the use of ultrasound. Ultrasound reduced both peak force and average force for all evaluations, but removing eggs treated with NNC was more difficult than removing eggs from dry, untreated hair (Fig. 3). In contrast, the conditionerbased ONC showed a trend for reduction of both forces.

\section{Participants}

At day 0 , we recorded baseline characteristics for all participants (Table 1). Most commonly, household sizes were 4 (57 participants), 6 (27 participants), and > 8 (16 participants), with households ranging from 2 to 12 members. For 110/134 (82.1\%) participants, one or more other family members took part, two families having six participants and one family five. In most households with multiple family members, there were participants in different treatment groups. The distribution of household size was similar between the groups (but was not tested statistically due to the non-independence of the data).

\section{Outcomes}

Of the 136 participants who gave consent, two, randomised to NNC, were mistakenly given $\mathrm{ONC}$, withdrawn, and excluded from ITT analysis. There were two withdrawals each from the NNC group, for lack of efficacy of the treatment, and the IPM/C group because of an unrelated adverse event. Each of these was included in the ITT analysis but excluded from the per-protocol (PP) analysis.

\section{Comparison of the lotion products}

In the comparison of lotion products, there were 103 evaluable participants, 50 treated using $\mathrm{NNC}$ and 53

Table 2 Comparison of the two lotion groups for the presence of lice and mean numbers of lice recovered

\begin{tabular}{|c|c|c|c|c|c|}
\hline \multirow[t]{2}{*}{ Endpoint } & \multirow[t]{2}{*}{ Group } & \multicolumn{4}{|l|}{ Day } \\
\hline & & 2 & 6 & 9 & 14 \\
\hline \multirow[t]{2}{*}{ Presence of lice } & NNC & $38.5 \%$ & $42.3 \%$ & $32.7 \%$ & $23.1 \%$ \\
\hline & IPM/C & $41.5 \%$ & $56.6 \%$ & $21.2 \%$ & $18.9 \%$ \\
\hline \multirow[t]{2}{*}{ Total lice } & NNC & 2.09 & 3.25 & 0.90 & 1.04 \\
\hline & IPM/C & 2.34 & 4.11 & 0.42 & 0.42 \\
\hline \multirow[t]{2}{*}{ Adult males } & NNC & 0.12 & 0.14 & 0.10 & 0.15 \\
\hline & IPM/C & 0.15 & 0.06 & 0.01 & 0.04 \\
\hline \multirow[t]{2}{*}{ Adult females } & NNC & 1.25 & 0.37 & 0.08 & 0.35 \\
\hline & IPM/C & 0.26 & 0.15 & 0.00 & 0.19 \\
\hline \multirow[t]{2}{*}{ Stage 3 nymphs } & NNC & 0.40 & 0.37 & 0.31 & 0.10 \\
\hline & IPM/C & 0.40 & 0.30 & 0.09 & 0.09 \\
\hline \multirow[t]{2}{*}{ Stage 2 nymphs } & NNC & 0.21 & 1.10 & 0.27 & 0.29 \\
\hline & IPM/C & 0.47 & 1.21 & 0.13 & 0.09 \\
\hline \multirow[t]{2}{*}{ Stage 1 nymphs } & NNC & 1.12 & 1.29 & 0.15 & 0.15 \\
\hline & IPM/C & 1.06 & 2.40 & 0.19 & 0.02 \\
\hline
\end{tabular}

There were no significant differences between the treatments with regard to numbers of lice found at any assessment day 
using IPM/C (Table 1). Analyses of the presence, number, and stages of lice from each assessment (Table 2) found no significant differences $(p<0.05)$ between the groups at any time point other than significantly $(p<0.05)$ more stage 1 nymphs in the NNC group at day 14. Fewer lice were found following the second treatment, but an increase occurred by day 14 from failure to kill all louse eggs.

The main analysis, the comparison of rates of cure, or cure followed by reinfestation, in the ITT population, found success in 36/50 (72.0\%) of those using NNC and $37 / 53(69.8 \%)$ treated with IPM/C. This difference was estimated as $2.2 \%$ (95\% confidence interval (CI), -15.4 to $19.7 \%$; odds ratio (OR) $1.112,95 \% \mathrm{CI}, 0.47$ to 2.61 ). There was no significant $(p<0.05)$ difference between the two treatments. Five cases of re-infestation after cure were found: four treated with NNC and one with IPM/ C. Of the 33 treatment failures (14 NNC vs. 16 IPM/C), two from each group had only stage 1 nymphs indicating ovicidal failure. For this analysis, the four dropouts were counted as treatment failures.
After the elimination of dropouts and participants failing to attend one of the post-treatment visits, the PP population was 94 , with successful treatments for $35 / 45$ $(77.8 \%)$ in the $\mathrm{NNC}$ group and $33 / 47(70.2 \%)$ in the IMP/C group (OR 1.4848, 95\% CI, 0.58 to 3.80 ).

Analyses of demographic subsets found no difference in the success rate in any of the subgroups analysed (Table 3). There was an overall non-significant trend for decreased efficacy on participants with heavier infestations, the exception being the within group difference in the rate of success for IPM/C where a light infestation was significantly $(p=0.01)$ more likely to be cured than a medium infestation. Cure rates also decreased slightly in both groups with increasing hair length.

Opinions of cosmetic characteristics were contradictory. Most (31/50, 62.0\%) thought NNC had a moderate/strong odour, compared with 11/53 (20.8\%) using IPM/C. Similar numbers, eight vs. seven respectively, reported the odour unpleasant, although 41/53 IPM/C users could not detect any odour, whereas NNC always

Table 3 Success rate for NNC and IPM/C by demographic characteristics subgroup

\begin{tabular}{|c|c|c|c|c|c|c|}
\hline \multirow[t]{2}{*}{ Subgroup } & & \multicolumn{2}{|l|}{$\mathrm{NNC}$} & \multicolumn{2}{|l|}{ IPM/C } & \multirow[b]{2}{*}{$p$ value } \\
\hline & & $n / N$ & $\%$ & $n / N$ & $\%$ & \\
\hline All participants & & $36 / 50$ & 72.0 & $37 / 53$ & 69.8 & NS \\
\hline \multirow[t]{2}{*}{ Sex } & Males & $10 / 12$ & 83.3 & $6 / 10$ & 60.0 & NS \\
\hline & Females & $26 / 38$ & 68.4 & $31 / 43$ & 72.1 & NS \\
\hline \multirow[t]{4}{*}{ Age } & 2 to 6 & $7 / 12$ & 58.3 & $9 / 11$ & 81.8 & NS \\
\hline & 7 to 9 & $11 / 19$ & 57.9 & $11 / 15$ & 73.3 & NS \\
\hline & 10 to 16 & $7 / 8$ & 87.5 & $10 / 16$ & 62.5 & NS \\
\hline & $>16$ & $11 / 11$ & 100 & $9 / 11$ & 81.8 & NS \\
\hline \multirow[t]{3}{*}{ Infestation $^{a}$} & Light & $26 / 33$ & 78.8 & $27 / 31$ & $87.1^{b}$ & NS \\
\hline & Moderate & $6 / 10$ & 60.0 & $5 / 11$ & $45.5^{\mathrm{b}}$ & NS \\
\hline & Heavy & $2 / 4$ & 50.0 & $5 / 8$ & 62.5 & NS \\
\hline \multirow[t]{4}{*}{ Hair length } & Close cut & $1 / 1$ & 100.0 & $2 / 2$ & 100 & NS \\
\hline & Above ears & $9 / 11$ & 81.8 & $4 / 6$ & 66.7 & NS \\
\hline & Ears to shoulders & $7 / 11$ & 63.6 & $10 / 13$ & 76.9 & NS \\
\hline & Below shoulders & $19 / 27$ & 70.4 & $22 / 32$ & 68.8 & NS \\
\hline \multirow[t]{3}{*}{ Hair thickness } & Fine & $6 / 7$ & 85.7 & $7 / 9$ & 77.8 & NS \\
\hline & Medium & $21 / 29$ & 72.4 & $17 / 19$ & 89.5 & NS \\
\hline & Thick & $9 / 14$ & 64.3 & $14 / 25$ & 65.0 & NS \\
\hline \multirow[t]{2}{*}{ Hair curl } & Straight & $21 / 31$ & 67.7 & $21 / 38$ & 55.3 & NS \\
\hline & Wavy or curly & $15 / 19$ & 78.9 & $13 / 15$ & 86.7 & NS \\
\hline \multirow[t]{2}{*}{ Hair type } & Normal & $34 / 49$ & 69.4 & $37 / 51$ & 72.6 & NS \\
\hline & Dry or oily & $0 / 1$ & 0.0 & $1 / 2$ & 50.0 & NS \\
\hline \multirow[t]{2}{*}{ Other family member in study } & No & $8 / 10$ & 80.0 & $7 / 9$ & 77.8 & NS \\
\hline & Yes & $26 / 40$ & 65.0 & $30 / 44$ & 68.2 & NS \\
\hline
\end{tabular}

${ }^{\mathrm{a}}$ Data missing from three participants in each of the treatment groups

'There was a significant difference in efficacy rates between "light" and "moderate" infestations given this treatment 
developed a strong onion-like odour as it dried. Significantly, more $(34 / 53,64.2 \%)(p<0.015)$ found IPM/C left the hair greasy after washing compared with 20/50 (40.0\%) using NNC.

\section{Comparison of treatments using ClearBrush ${ }^{\circledast}$}

ClearBrush $^{\circ}$ combing with ultrasound examined $17^{2}$ people treated with NNC and 14 with ONC. On days 0 and 7, the outcomes were analysed for the presence of lice and louse eggs/nits, but for each of days 2, 6, 9 , and 14, the analysis was for the presence of live lice only. The Clearbrush ${ }^{\circ}$ did not remove lice or nits from two participants using $\mathrm{ONC}$ and one using NNC, but all other assessment data were complete. Table 4 shows the percentage of participants with lice and mean number of each development stage, and any significant differences, at each of the 6 days.

The ClearBrush ${ }^{\circ}$-treated groups showed significantly $(p \leq 0.05)$ more stage 1 and 2 nymphs on ONC users at day 6 and stage 3 nymphs at days 6 and 14. The ONC group had significantly $(p<0.05)$ more lice in total at day 9 , becoming highly significant $(p<0.005)$ by day 14. Although ONC removed more than 13 times as many nits on day 0 and over six times the number on day 7, this difference was not significant, due partly to the small group sizes but also the skewed distribution of nits on a few people.

The overall efficacy comparison between NNC and ONC showed a significant $(p<0.01)$ pediculicidal advantage for the neem-in-silicone treatment (NNC), although the group sizes were too small for firm conclusions. NNC was successful for 14/17 (82.4\%) compared with 4/14 (28.6\%) cures for ONC (OR 11.67; $95 \%$ CI 2.13 to 64.04). No differences were found for efficiency of removing nits because so many cases had few nits present, but NNC plus ClearBrush $^{\circ}$ showed a slight non-significant trend $(p=0.53)$ in favour of using the comb as an adjunct to treatment.

\section{Adverse events}

There were seven reported adverse events in five people. None was linked with treatment, although one cough was possibly exacerbated by the odour of neem. All but one of the events, back pain, were associated with seasonal respiratory tract infections. One girl in the IPM/C group experienced two adverse events, one of which was an asthma attack requiring a visit to hospital for nebulisation,

Table 4 Comparison of the two ultrasound comb-treated groups for the presence of lice, presence of louse egg/nits, and mean numbers of lice and louse eggs/nits recovered

\begin{tabular}{|c|c|c|c|c|c|c|c|}
\hline \multirow[t]{2}{*}{ Endpoint } & \multirow{2}{*}{$\begin{array}{l}\text { Treatment } \\
\text { Lotion }\end{array}$} & \multicolumn{6}{|c|}{ Day of study } \\
\hline & & 0 & 2 & 6 & 7 & 9 & 14 \\
\hline \multirow[t]{2}{*}{ Presence of live lice } & NNC & $94.1 \%$ & $35.3 \%$ & $41.2 \%$ & $32.7 \%$ & $11.8 \%$ & $17.7 \%$ \\
\hline & ONC & $85.7 \%$ & $64.3 \%$ & $71.4 \%$ & $21.2 \%$ & $50.0 \%$ & $71.4 \% *$ \\
\hline \multirow[t]{2}{*}{ Total lice } & NNC & 20.9 & 2.12 & 2.06 & 0.90 & 0.12 & 0.35 \\
\hline & ONC & 28.4 & 4.93 & 11.86 & 0.42 & $1.79 * *$ & 5.93 \\
\hline \multirow[t]{2}{*}{ Adult males } & NNC & 1.69 & 0.53 & 0.06 & 0.38 & 0.0 & 0.06 \\
\hline & ONC & 2.25 & 0.29 & 0.79 & 0.33 & 0.07 & 0.86 \\
\hline \multirow[t]{2}{*}{ Adult females } & NNC & 2.69 & 0.65 & 0.18 & 0.06 & 0.0 & 0.18 \\
\hline & ONC & 3.50 & 0.86 & 1.21 & 0.75 & 0.14 & 1.79 \\
\hline \multirow[t]{2}{*}{ Stage 3 nymphs } & NNC & 3.31 & 0.59 & 0.18 & 0.13 & 0.0 & 0.12 \\
\hline & ONC & 3.33 & 0.29 & $1.71^{* *}$ & 1.67 & 0.29 & $1.00^{* *}$ \\
\hline \multirow[t]{2}{*}{ Stage 2 nymphs } & NNC & 6.00 & 0.18 & 0.59 & 1.81 & 0.06 & 0.0 \\
\hline & ONC & 4.33 & 1.14 & $3.07^{* *}$ & 6.17 & 1.00 & 1.21 \\
\hline \multirow[t]{2}{*}{ Stage 1 nymphs } & NNC & 7.25 & 0.18 & 1.06 & 5.94 & 0.06 & 0.0 \\
\hline & ONC & 13.7 & 2.36 & $5.07^{* * *}$ & 12.8 & 0.29 & 1.07 \\
\hline \multirow[t]{2}{*}{ Presence of eggs/nits } & NNC & $94.1 \%$ & - & - & $82.4 \%$ & - & - \\
\hline & ONC & $71.4 \%$ & - & - & $78.6 \%$ & - & - \\
\hline \multirow[t]{2}{*}{ Number of eggs/nits } & NNC & 13.25 & - & - & 19.80 & - & - \\
\hline & ONC & 181.83 & - & - & 124.20 & - & - \\
\hline
\end{tabular}

Mean figures should only be taken as indicative due to the skew distribution of the data 


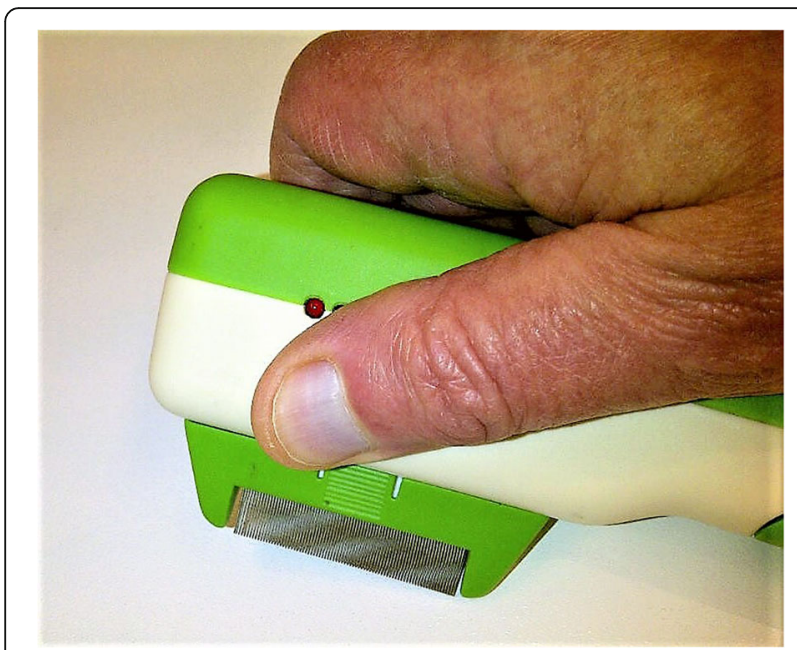

Fig. 4 Hand position taken up by investigators, close to the tooth unit, in order to retain a firm grip whilst combing. Moving the hand to this position largely eliminated the angle of the combing head, which had previously been shown to improve louse egg removal. Consequently, this would have resulted in some of the extra combing efficiency delivered through the ultrasound actuation being lost in practice

resulted in her dropping out to avoid further possible stress despite no evidence of a link between treatment on day 0 and the attack on day 5 . There was no difference between groups in respect of frequency, outcome, action taken, relationship to study treatment, or severity.

\section{Discussion}

This clinical investigation showed that a silicone fluid formulated with a heavy plant oil, in this case neem seed oil, can be a pediculicide as effective as other lipid and silicone mixes. However, we detected no activity from the neem oil apart from physical occlusion effects. The activity of the solvent showed in the relative performance of the silicone-based lotion ( $74.6 \%$ success overall) and original Nice ' $\mathrm{n}$ Clear head lice lotion (28.6\% success), which was equally ineffective in an earlier study (Brown and Burgess 2017).

Irrespective of activity, the natural oil presents a number of practical problems. Neem seed oil is often cloudy, difficult to clarify by filtration, becomes gelatinous at cool room temperatures, and hard to formulate in any dosage form other than an emulsion, trapping the oil in micelles. In the silicone base, only one of several apparently similar PEG/PPG-dimeticone copolymer formulation aids allowed the mix to remain physically stable. A major problem was the recurrence of turbidity, apparently due to oxidative interactions with the air. It was difficult to wash out from hair requiring two or three shampoo washes, although the isopropyl myristate in IPM/C was even more difficult to remove, especially from fine hair. However, the most important drawback was the pungent odour, which is was not masked by addition of a citronella fragrance.

Clinically, the neem-silicone lotion (NNC) with the ultrasound generating ClearBrush ${ }^{\bullet}$ was non-significantly better than lotion alone but less successful at removing nits than the comb with the original lotion (ONC), although the skew distribution of eggs among relatively few participants meant the results were inconclusive. The benefits of using a comb with an angled head were demonstrated in laboratory tests (Burgess et al. 2016) but not replicated clinically. The prototype was not ergonomic with a long, thick handle to accommodate batteries, which required effort to pull it through the hair. Consequently, to retain a firm grip, investigators often held it around the tooth unit (Fig. 4), which negated the angle of the combing head. A design change would be required to address the problem.

In some ways, using the ultrasound comb was less physically demanding because the cavitation effects predicted to facilitate penetration of fluid into the spaces between the egg glue and the hairs themselves (Fig. 5) made sliding easier, especially with the ONC conditioner

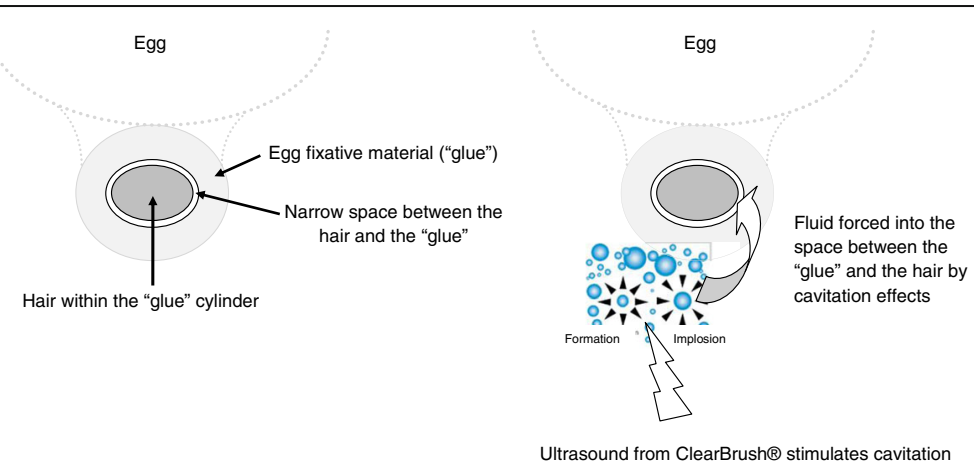

Fig. 5 Diagrammatic representation of the putative effect of ultrasound to produce cavitation effects in fluids resulting in increased flow into the space between the hair and the fixative "glue" binding the louse egg to the hair 
lotion. In contrast, when using the silicone-based lotion, the comb scraped over the eggshells, which were not removed as efficiently.

\section{Conclusions}

As previously (Brown and Burgess 2017), this project could not identify any specific activity for neem in either formulation. The ClearBrush ${ }^{\bullet}$ device concept has also not been confirmed clinically and not exploited commercially, requiring a more compact power unit to make the device small enough to fit into the hand. Nevertheless, this project has demonstrated scope for improvement in treating head louse infestation and nit removal suggesting further investigation of the principles could prove beneficial.

\begin{abstract}
Abbreviations
CB: ClearBrush ${ }^{\oplus}$ ultrasonic nit comb; Cl: Confidence interval; IPM/C: 50:50 isopropyl myristate in cyclomethicone; ITT: Intention to treat; $n$ : Number of participants with a particular characteristic; $N$ : Total number of participants in a particular cohort group; NNC: Reformulated Nice ' $n$ Clear head louse lotion; ONC: Original formulation Nice ' $n$ Clear head lice lotion; OR: Odds ratio; PEG/ PPG: Polyethylene glycol/polypropylene glycol; PP: Per-protocol
\end{abstract}

\section{Acknowledgements}

The project co-ordinator was A Nelson \& Co Ltd. (UK). Other SME partners involved were Denman International Ltd. (UK) and Innowacja Polska Sp. z.o.o. (Poland). Thanks are due to the following individuals for their contributions: Ulli Jonsson and Peter Palmer (Nelsons-coordinators), Andrew Harrower and Marcus McCay (Denman — prototype comb design and production), and Marcin Opoka and Leonard Płonka (Innowacja Polska—ultrasound unit design and technical co-ordination). Sample size calculations were performed on behalf of the sponsor by PN Lee Statistics and Computing Ltd., an independent statistical consultancy. In addition to the authors, treatments and/or assessments were performed by Audrey Pepperman, Christine Sullivan, Geraldine Matlock, Gillian Clarke, and lan M Jones. Dr. Paul Silverston was the research physician and evaluated the adverse events. The documents and conduct of Good Clinical Practice were monitored by Ulli Jonsson on behalf of A Nelson \& Co Ltd.

\section{Funding}

This project was part of a co-operative research project initiated under the Sixth Framework Programme of the European Commission, project number COOP-CT-2005-017916, with the title "A novel integrated ultrasonic brush and sonically activated lotion to provide a full system approach to the eradication of the European head louse menace". The project co-ordinator was A Nelson \& Co Ltd. (UK) who played no role in the study design, its execution, interpretation of the data, decision to publish, or the preparation of the manuscript.

\section{Availability of data and materials}

The datasets used and/or analysed during the current study are available from the corresponding author on a reasonable request.

\section{Authors' contributions}

IFB, ERB, and NAB collectively conceived and designed the clinical investigation, organised the study, performed the treatments, and collected the raw data. IFB performed the analyses of the data and wrote the draft manuscript. MNB designed and performed the laboratory experiments for evaluation of the ClearBrush ${ }^{\ominus}$. All authors read and approved the final manuscript.

\section{Ethics approval and consent to participate}

The study complied with Good Clinical Practices and the principles of the Declaration of Helsinki and of European Union Directive 2001/20/EC. Participants received study information at least $24 \mathrm{~h}$ before enrolment and stated that they understood its purpose and requirements before giving consent. Parents/guardians gave consent for children younger than 16 years, and capable children provided written assent witnessed by the parent/ guardian. Ethical approval was granted by Huntingdon Local Research Ethics Committee (application number 07/Q0104/44).

\section{Consent for publication}

Not applicable.

\section{Competing interests}

IFB has been a consultant to various makers of pharmaceutical products, medical devices, and combs for treating infestations of head lice and their eggs. The other authors declare that they have no competing interests.

\section{Publisher's Note}

Springer Nature remains neutral with regard to jurisdictional claims in published maps and institutional affiliations.

Received: 23 March 2017 Accepted: 20 September 2017

Published online: 03 November 2017

\section{References}

Abdel-Ghaffar F, Al-Quraishy S, Al-Rasheid KA, Mehlhorn H. Efficacy of a single treatment of head lice with a neem seed extract: an in vivo and in vitro study on nits and motile stages. Parasitol Res. 2012;110:277-80.

Abdel-Ghaffar F, Semmler M. Efficacy of neem seed extract shampoo on head lice of naturally infected humans in Egypt. Parasitol Res. 2007;100:329-32.

Barker SC, Altman PM. A randomised, assessor blind, parallel group comparative efficacy trial of three products for the treatment of head lice in children — melaleuca oil and lavender oil, pyrethrins and piperonyl butoxide, and a "suffocation" product. BMC Dermatol. 2010;10:6.

Brown CM, Burgess IF. Can neem oil help eliminate lice? Randomised controlled trial with and without louse combing. Adv Pediatr Res. 2017:4:9.

Burgess IF. Do nit removal formulations and other treatments loosen head louse eggs and nits from hair? Med Vet Entomol. 2010;24:55-61.

Burgess IF, Brunton ER, Burgess NA. Clinical trial showing superiority of a coconut and anise spray over permethrin $0.43 \%$ lotion for head louse infestation, ISRCTN9646978. Eur J Pediatr. 2010;169:55-62.

Burgess IF, Lee PN, Matlock G. Randomised, controlled, assessor blind trial comparing $4 \%$ dimeticone lotion with $0.5 \%$ malathion liquid for head louse infestation. PLoS One. 2007:2:11.

Burgess MN, Brunton ER, Burgess IF. A novel concept nit comb using ultrasound actuation: pre-clinical evaluation. J Med Entomol. 2016;53:152-6.

Dallal GE. Randomization.com. 2007. http://www.randomization.com. Accessed 11 Sept 2007

de Souza BV, de Oliveira GL, de Oliveira NJ, da Silva Ribeiro DC. Estudo comparativo da eficiência de três diferentes pentes finos na retirada de piolhos e lêndeas. Rev Bras Med. 2001;58:398-402.

Grieve KA, Altman PM, Rowe SJ, Staton JA, Oppenheim VMJ. A randomised, double-blind, comparative efficacy trial of three head lice treatment options: malathion, pyrethrins with piperonyl butoxide and MOOV head lice solution. Aust Pharmacist. 2007;26:738-43.

Heukelbach J, Oliveira FA, Speare R. A new shampoo based on neem (Azadirachta indica) is highly effective against head lice in vitro. Parasitol Res. 2006;99:353-6.

Mehlhorn H, Abdel-Ghaffar F, Al-Rasheid KA, Schmidt J, Semmler M. Ovicidal effects of a neem seed extract preparation on eggs of body and head lice. Parasitol Res. 2011;109:1299-302.

Schmahl G, Al-Rasheid KA, Abdel-Ghaffar F, Klimpel S, Mehlhorn H. The efficacy of neem seed extracts (Tre-san ${ }^{\oplus}$, MiteStop ${ }^{\oplus}$ ) on a broad spectrum of pests and parasites. Parasitol Res. 2010;107:261-9.

Thawornchaisit P, Amornsak W, Mahannop P, Buddhirakkul P, Pandii W, Connellan $\mathrm{P}$, et al. Combined neem oil $6 \% \mathrm{w} / \mathrm{w}$ and eucalyptus oil $16 \% \mathrm{w} / \mathrm{w}$ lotion for treating head lice: in vitro and in vivo efficacy studies. J Pharm Pract Res. 2012:42:189-92. 